\title{
Study of the influence of properties of dusty ferromagnetic additives on the increase of cement activity
}

\author{
Serhiy Sakhno ${ }^{1, *}$, Lyudmyla Yanova $^{2}$, Olena Pischikova ${ }^{2}$, and Serhii Chukharev ${ }^{3}$ \\ ${ }^{1}$ Kryvyi Rih National University, Civil Engineering Faculty, 11 Vitalii Matusevych Str., Kryvyi Rih, Ukraine \\ ${ }^{2}$ Kryvyi Rih National University, Mining and Metallurgical Faculty, 11 Vitalii Matusevych Str., Kryvyi Rih, Ukraine \\ ${ }^{3}$ National University of Water and Environmental Engineering, Department of Mining Development and Mining, 11 Soborna Str., \\ Rivne, Ukraine
}

\begin{abstract}
Sustainable development of construction materials is directly related to research on the processes of hydration of binders. Builders need better types of cement, with lower cost and energy consumption in production. The development of spin chemistry methods allows us to consider the processes of hydration and structure formation of binders from the spin state of the elements involved in chemical reactions. Magnetic interactions have a significant effect on the spin dynamics and the control of the spin multiplicity of radical pairs. The practical implementation of magnetic effects on a binder can be carried out in various ways. However, a long-term impact can be achieved only by introducing ferromagnetic substances into the binders. In the paper presented the results of a study of the influence of the characteristics of finely dispersed powdered ferromagnetic additives on the strength characteristics of cement. Ferromagnetic additives regulate the behavior of the reactants during rotation during the hydration of the binders due to magnetic interactions and control the reactivity of the chemical reaction. A comparative analysis revealed that it is most expedient to use as powdery ferromagnetic additives are the waste from mining and processing enterprises of the Krivorozhsky field. The work investigated the magnetic and dispersed characteristics of 12 different dust. The experiments showed that the origin of dust and the method of their capture are determined their magnetic characteristics. Preparation of samples with the dust and determination of the strength characteristics of cement were carried out by standard methods. The results obtained made it possible to reveal the laws of the effect of the dispersed and magnetic properties of various dust on the degree of activation of binders.
\end{abstract}

\section{The problem and its relationship with scientific and practical tasks}

Cement is one of the most essential currently used building materials. The main components for the production of cement are clay and limestone. In the process of co-firing limestone and clay, a clinker obtained consisting of four primary minerals: alite, belite, tricalcium aluminate, and tetracalcium alumino ferrite. Cement is a product of joint grinding of clinker and gypsum, which introduced to regulate the setting time.

After mixing the cement with water, is formed a cement paste, which begins to harden after 40 minutes and finally turns into a cement stone after 6 hours. Cement hardening occurs due to hydrolysis reactions and subsequent hydration of its components. After the influence of water on the surface of cement formed particles of calcium hydroxide, calcium silicate hydrate, and calcium aluminate hydrate. In the process of hardening, neoplasms pass from a colloidal state to a fine crystalline one. Microcrystals gradually grow together. The processes of gel formation, crystallization, and compaction lead to the transformation of cement paste into an artificial high-strength stone material.

The currently existing theories of hardening binders (A. Le Chatelier, V. Michaelis, A. A. Baykov, and others.) primarily develop physicochemical ideas about the mechanism of formation of hydrated compounds and hardening of mixtures of binders with water when they interact with each other. Moreover, the interpretation of the processes occurring during the hardening of the cement paste is based solely on charges. It assumed that during the solidification of binders, the forces of electrostatic nature are decisive since the particles of the new phase are not neutral. Because of the orientation of the dipoles of the dispersion medium adsorbed on their surface, they acquire a particular charge.

Despite numerous studies, this approach has not yet been able to reveal the true nature of the forces leading to the strengthening of the structure and the synthesis of the strength of cement stone and to create a unified and universally accepted theory of hardening of cementitious systems.

\footnotetext{
* Corresponding author: budfac@gmail.com
} 


\section{Analysis of research and publications}

The intensive development of spin chemistry methods allowed us to consider the processes of hydration and structure formation of binders in juxtaposition with the spin state of the elements involved in chemical reactions [1]. In [2], were presented experimental results that prove the presence of radical reactions during the hardening of the cement. In $[1,2]$ was shown that cement is paramagnetic substances, and the appearance of paramagnetic centers in them explained by the appearance of spin-orbitals populated by a single electron in an atomic or molecular system. The method of electron paramagnetic resonance made it possible to quantify the paramagnetic properties of cement and their change during hardening.

In [5] was shown that the number of paramagnetic centers in cement increases up to 3 days of hardening and then decreases to a level below the initial value. According to the results of studies $[3,4]$, the concentration of paramagnetic centers in cement is high and can reach $1022 \mathrm{spin} / \mathrm{g}$. The authors found that cement with a higher concentration of paramagnetic centers has higher strength characteristics. Research [5] has shown that cement hardens due to the recombination of paramagnetic centers, which leads to the formation of new phase states, the presence of which contributes to the hardening of the cement mortar and the creation of cement stone.

In [6] was shown that magnetic interactions have a significant influence on the spin dynamics and the control of the spin multiplicity of radical pairs. In this case, magnetic interactions, without affecting the chemical reaction itself, switch it from spin-forbidden channels to spin-allowed ones, regulating the spin behavior of the reagents, replacing non-reaction channels with reaction ones. [6]. Thereby, the reactivity of the chemical reaction is regulated.

It can be argued that magnetic fields can affect the chemical processes that occur during the hydration of binders because of the presence of radical reactions during the hardening of the cement.

Previously it was researched the magnetic activation of water for binder [7], activation of a binder by magnetic treatment during grinding [8], and in the interval between grinding and mixing the binder with water [9].

The method of activation by magnetizing the binder itself $[8,10-12]$ has the disadvantage that.

In most cases have low magnetizability, which also lasts a relatively short time. Thus, this method has a weak effect on the course of hardening reactions of binders.

A method for activating a binder by magnetizing a cement paste has developed in many embodiments. For activation, fields of various intensities were used, as well as variable and pulsating fields. Of all known, this method gives the best results [10] due to the relatively more powerful magnetic fields superimposed on the hardening mixture, as well as the stability of these fields. However, it also has drawbacks that affect the strength characteristics of the binder. First of all, this is a short time of exposure to magnetic fields on the binder. In most cases, it does not exceed 15 to 30 minutes, and this is only a small fraction of the total binder curing time.
Accordingly, the effect of activation of the binder decreases.

In addition to the above disadvantages, all of the above methods of activating binders have one more. The binder can be activated only at the place of preparation of concrete or mortar. At the same time, the manufacturing technology of building products is complicated and changed, the time spent on manufacturing specific products increases, the need for additional equipment and specially trained personnel arises, and the likelihood of errors in choosing the magnitude of the magnetic field strength, the time of its exposure and the dosage of additional components increases.

\section{Formulation of the problem}

The described disadvantages are absent if the binders activated by introducing into their composition a premagnetized ferromagnetic powder. The effectiveness of this method because by the fact that the influence of magnetic fields occurs at all stages of the creation of a binder and its hardening, starting from co-grinding cement with powder and ending with long hardening periods of 1 year or more. In this case, each stage contributes to the increase in strength. It is advisable to use powdered ferromagnetic production waste as additives to reduce the cost of activation of binders. As such substances, we proposed the use of dust generated at various stages of iron ore enrichment. At the same time, this allows the disposal of dusty waste from mining and processing plants to sustain the environmental situation. In table 1 is given the characteristics of the enrichment products of various deposits.

Table 1. Characterization of the magnetic properties of concentrates obtained from ores of various deposits [13, 14].

\begin{tabular}{|c|c|c|c|}
\hline \multirow{2}{*}{ Sample } & \multirow{2}{*}{$\begin{array}{c}\text { Density, } \\
-10^{3} \\
\mathbf{k g} / \mathbf{m}^{3}\end{array}$} & \multicolumn{2}{|c|}{$\begin{array}{l}\text { Magnetization, } \\
-10^{3}, \mathbf{A} / \mathbf{m}\end{array}$} \\
\hline & & Maximum & Residual \\
\hline $\begin{array}{l}\text { Magnetite of the Akkerman } \\
\text { field }\end{array}$ & 4.2 & 45.7 & 23.0 \\
\hline $\begin{array}{l}\text { Magnetite of the } \\
\text { Krivorozhsky deposit }\end{array}$ & 4.7 & 189.0 & 60.5 \\
\hline $\begin{array}{l}\text { Maghemite of the Vyatka } \\
\text { field }\end{array}$ & 4.0 & 119.0 & 42.5 \\
\hline $\begin{array}{l}\text { Magnetite of the } \\
\text { Vysokogorsky deposit }\end{array}$ & 4.8 & 196.0 & 22.0 \\
\hline $\begin{array}{l}\text { Magnetite of the Lebedinsky } \\
\text { deposit }\end{array}$ & - & 80.5 & 20.0 \\
\hline $\begin{array}{l}\text { Magnetite of the Tashtagol } \\
\text { deposit }\end{array}$ & - & 64.5 & 12.5 \\
\hline $\begin{array}{l}\text { Magnetite of the } \\
\text { Kremenchuk field }\end{array}$ & - & 72.0 & 22.3 \\
\hline $\begin{array}{l}\text { Magnetite of the Sokolovsky } \\
\text { deposit }\end{array}$ & - & 105.4 & - \\
\hline $\begin{array}{l}\text { Magnetite of the } \\
\text { Kachkanarsky deposit }\end{array}$ & - & 126.0 & 37.0 \\
\hline
\end{tabular}

The table data shows that the largest residual magnetization has the magnetite of the Krivorozhsky deposit. Therefore, the most effective will be the addition of dusty wastes of this field.

In table 2 is shown the chemical and mineral 
composition of the dust concentrate from the mining and processing enterprises of Krivbass.

After agglomeration and pelletizing of the concentrate in the dust, the amount of bound calcium oxide and carbon increases. In table 3 is shown the chemical composition of the sinter dust.

Table 2. Chemical and mineral composition of the dust of concentrate of mining and processing plants of Krivorozhsky deposit $[13,14]$.

\begin{tabular}{|c|c|c|}
\hline \multirow{4}{*}{$\begin{array}{c}\text { Chemical } \\
\text { composition, \% }\end{array}$} & Components & Content \\
\cline { 2 - 3 } & $\mathrm{Fe}$ & 65.2 \\
\cline { 2 - 3 } & $\mathrm{SiO}_{2}$ & 8.17 \\
\cline { 2 - 3 } & $\mathrm{Al}_{2} \mathrm{O}_{3}$ & 0.15 \\
\cline { 2 - 3 } & $\mathrm{CaO}$ & 0.11 \\
\cline { 2 - 3 } & $\mathrm{MgO}$ & 0.4 \\
\cline { 2 - 3 } & $\mathrm{P}$ & 0.01 \\
\hline \multirow{2}{*}{$\begin{array}{c}\text { Mineral } \\
\text { composition, \% }\end{array}$} & $\mathrm{S}$ & 0.014 \\
\cline { 2 - 3 } & other & 0.8 \\
\cline { 2 - 3 } & magnetite & 64.3 \\
\hline
\end{tabular}

Table 3. The chemical composition of the dust of the sinter machine [15].

\begin{tabular}{|c|c|}
\hline Components & Content, \% \\
\hline $\mathrm{FeO}$ & $9-19$ \\
\hline $\mathrm{Fe}_{2} \mathrm{O}_{3}$ & $45-60$ \\
\hline $\mathrm{SiO}_{2}$ & $6.5-10$ \\
\hline $\mathrm{Al}_{2} \mathrm{O}_{3}$ & $0.5-1.5$ \\
\hline $\mathrm{CaO}$ & $6.3-9$ \\
\hline $\mathrm{MgO}$ & $0.5-1.5$ \\
\hline $\mathrm{MnO}$ & $0.2-0.3$ \\
\hline $\mathrm{P}_{2} \mathrm{O}_{5}$ & $0.03-0.05$ \\
\hline $\mathrm{S}$ & $0.2-0.5$ \\
\hline
\end{tabular}

In table 4 is given the dispersed composition of the dust.

Table 4. The dispersed composition of the dust of mining and processing plants.

\begin{tabular}{|c|c|}
\hline Particle diameter, $\boldsymbol{\mu m}$ & Particle mass, \%o \\
\hline 4 & $3.2-5.1$ \\
\hline $4-6.3$ & $2.1-2.3$ \\
\hline $6.3-10$ & $4-5$ \\
\hline $10-16$ & $8.3-8.7$ \\
\hline $16-25$ & $10-12$ \\
\hline $25-40$ & $13-15$ \\
\hline $40-63$ & $12-15$ \\
\hline $63-100$ & $20-22.5$ \\
\hline $100-400$ & $14.3-16.2$ \\
\hline
\end{tabular}

Research under a microscope shows that particles of iron ore dust are irregular in shape with sharp edges. Most particles have color from brown to black. In the total mass, depending on the stage of enrichment, the color of the dust changes from red to black.

The highest dust formation occurs at the following stages of enrichment: during magnetizing roasting of the ore, during transportation of concentrate, during roasting of pellets, during agglomeration of the concentrate.

Because this, did study four dust groups:
- dust generated by magnetizing roasting of the ore;

- dust generated during transportation of the concentrate;

- dust generated during the firing of pellets;

- dust formed during the agglomeration of the concentrate.

Dust generated after magnetizing roasting of ore during transportation of concentrate and roasting pellets is captured either in cyclones or in an aqueous medium. During dust collection on dust are not superimposed electromagnetic fields. For a complete study of the properties of these dust, it proposed to study them in an unmagnified state and magnetized in a constant magnetic field with a strength of $75 \mathrm{kA} / \mathrm{m}$.

The dust collection of sinter production carried out in electrostatic precipitators. During the capture process, on this type of dust are superimposed high-intensity electromagnetic fields. Given this, the determination of the dust properties of sinter production carried out without additional magnetic treatment.

Each type of dust is assigned a number, which later used on the figures of this article. In table 5 is given the name of dust and their ID number.

Table 5. The identification numbers of different types of dust.

\begin{tabular}{|c|c|}
\hline $\begin{array}{l}\text { Dust ID } \\
\text { number }\end{array}$ & Type of dust \\
\hline 1 & $\begin{array}{l}\text { not magnetized dust from the collector generated } \\
\text { by magnetizing roasting of the ore }\end{array}$ \\
\hline 2 & $\begin{array}{l}\text { magnetized dust from the collector generated by } \\
\text { magnetizing roasting of the ore }\end{array}$ \\
\hline 3 & $\begin{array}{l}\text { not magnetized dust from the scrubber generated } \\
\text { by magnetizing roasting of the ore }\end{array}$ \\
\hline 4 & $\begin{array}{l}\text { magnetized dust from the scrubber generated by } \\
\text { magnetizing roasting of the ore }\end{array}$ \\
\hline 5 & not magnetized dust of concentrate \\
\hline 6 & magnetized dust of concentrate \\
\hline 7 & $\begin{array}{l}\text { not magnetized dust generated during the firing } \\
\text { of pellets }\end{array}$ \\
\hline 8 & $\begin{array}{l}\text { magnetized dust generated during the firing of } \\
\text { pellets }\end{array}$ \\
\hline 9 & the dust of the sintering machine from cyclone \\
\hline 10 & \begin{tabular}{|l} 
the dust of the sintering machine from \\
electrostatic precipitator zone I
\end{tabular} \\
\hline 11 & $\begin{array}{l}\text { the dust of the sintering machine from } \\
\text { electrostatic precipitator zone II }\end{array}$ \\
\hline 12 & $\begin{array}{l}\text { the dust of the sintering machine from } \\
\text { electrostatic precipitator zone III }\end{array}$ \\
\hline
\end{tabular}

\section{Statement of material and results}

The dust research methodology includes the following components: sampling and preparation methods and methods for determining dust properties.

Sampling and preparation of samples carried out according to the following procedure.

A shovel of non-magnetic material used to extract 20 $\mathrm{kg}$ of dust from industrial dust collectors. Each sample was placed in its plastic bag and then in an aluminum tank. Then the sample was transported to the laboratory. Further processing of dust was carried out far from sources of electromagnetic radiation.

In the laboratory, the sample was thoroughly mixed and then quartered. Stirring and quartering continued until 
the amount of material in the sample were reduced to $100 \mathrm{~g}$. On a sheet of clean white paper of $350 \times 350 \mathrm{~mm}$ in size, the sample was mixed and divided into ten equal parts using a plastic spatula.

In one of the formed parts of the dust was immersed a glass rod pre-moistened with glycerin. Then, the stick with adhering dust was placed in a cell with glycerin and mixed until was obtained a homogeneous suspension. Similarly, it was prepared suspensions from the remaining parts of the dust. Each cuvette was alternately placed in the installation to study the properties of dust.

Dust studied in a specially designed setup (Fig. 1) that consists of a digital microscope and a system of electromagnets mounted on a stage. An energy source feeds the orientation coils, as well as the illuminator time relay, so the control circuit provides the simultaneous switching on and off of the coil measurement system and the electronic stopwatch.

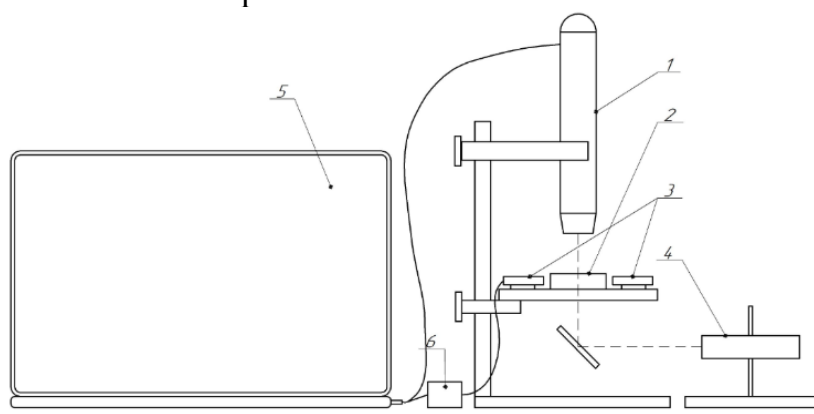

Fig. 1. Installation for determining the magnetic and dispersed characteristics of additives. 1 - digital microscope; 2 - a cuvette with glycerin and dust; 3 - orientation coil system; 5 laptop with control program; 6 - the digital coil of the power control unit.

A cuvette (Fig. 1) with glycerin and test dust was placed under the microscope lens, after which the orientation system of the coils turned on. In this case, a uniform magnetic field is created in the cuvette, orienting the magnetic particles. Then, using a time relay, the measuring system of coils and the electronic stopwatch are simultaneously switched on. After a particular time, the system turns off.

The rotation angle of all particles is fixed on the photograph at the same speed to eliminate the error of visual observation. First is photographed the arrangement of particles oriented perpendicular to the measuring field along the orientation. Then, using the time relay, the measuring field is switched on for a while and is photographed a new arrangement of particles. Two photographs determine the angle of rotation of particles $\beta$, rotation time $t$, and particle sizes, which makes it possible to decide on magnetic characteristics (Fig. 2). From each sample are determined the parameters of at least one hundred particles.

In the research of dust were determined the fractional composition, the fraction of dust particles with magnetic properties, the average magnetic moment of particles of one fraction, and the average magnetization of all dust.

The dispersed composition was determined by the following fractions: less than $1.4 \mu \mathrm{m} ; 1.4-4.2 \mu \mathrm{m} ; 4.2-$ $7.0 \mu \mathrm{m} ; 12.6-15.6 \mu \mathrm{m} ; 15.6-31.2 \mu \mathrm{m} ; 31.2-46.8 \mu \mathrm{m}$; $46.8-62.0 \mu \mathrm{m}$; more than $62.0 \mu \mathrm{m}$.

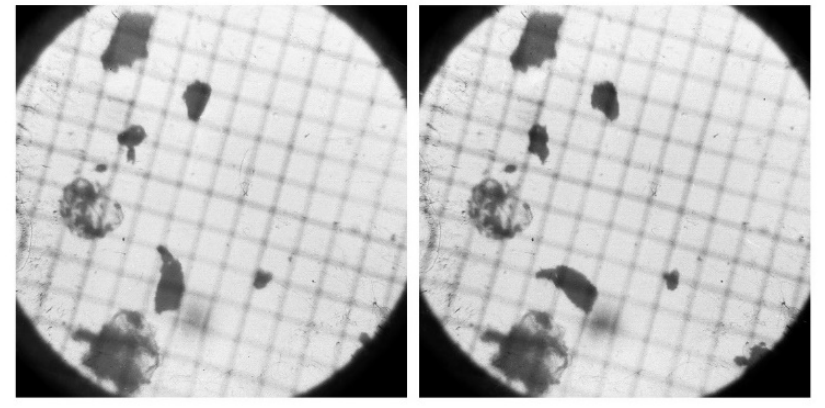

Fig. 2. Dust in the installation for determining magnetic and dispersed characteristics: a) particles in the orientation field; b) particles in the measuring field.

The studies of the characteristics of the dust of magnetizing roasting of the ore used dust taken from the collector during preventive stops of kilns and dust made from the scrubber in the form of a suspension in water. The suspension was precipitated and dried at a temperature of $105-110^{\circ} \mathrm{C}$ to constant weight.

In Fig. 3 and 4 show the disperse and magnetic properties of the dust from kilns taken from the collector, and in Fig. 5 and 6 taken from the scrubber.

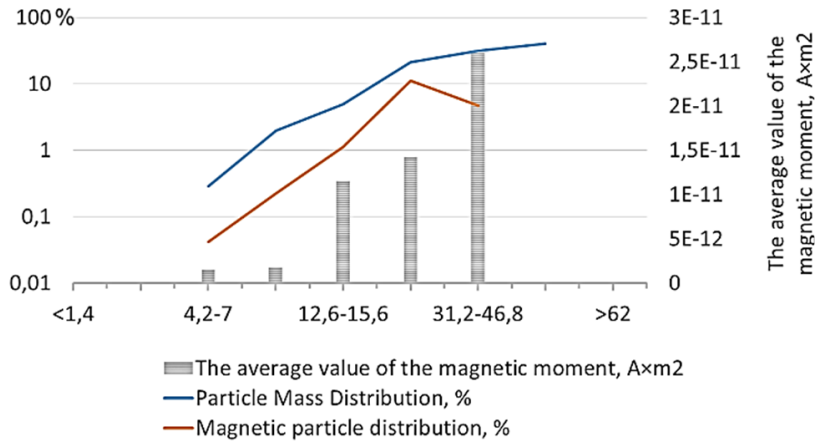

Fig. 3. The disperse and magnetic properties of the not magnetized dust from the collector generated by magnetizing roasting of the ore

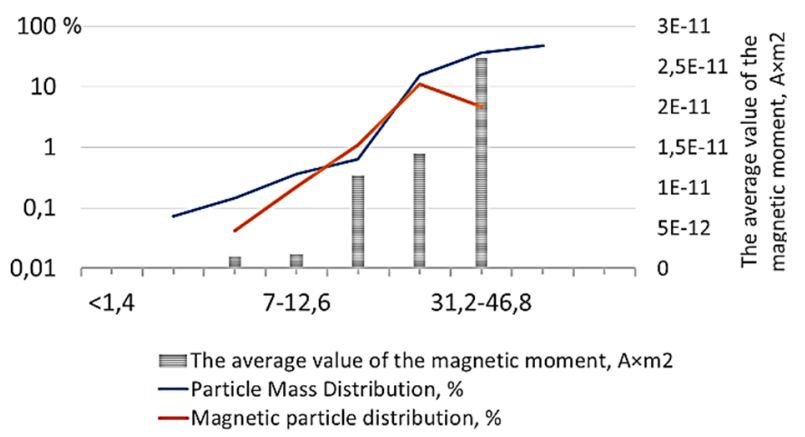

Fig. 4. The disperse and magnetic properties of the magnetized dust from the collector generated by magnetizing roasting of the ore

A comparative analysis of the results shows that the dust taken from the collector has a more significant number of large fractions than the dust made from the scrubber. In the collector, predominantly large particles settle, which are more challenging to carry through the air stream. The dust taken from the collector has an initial magnetization. 


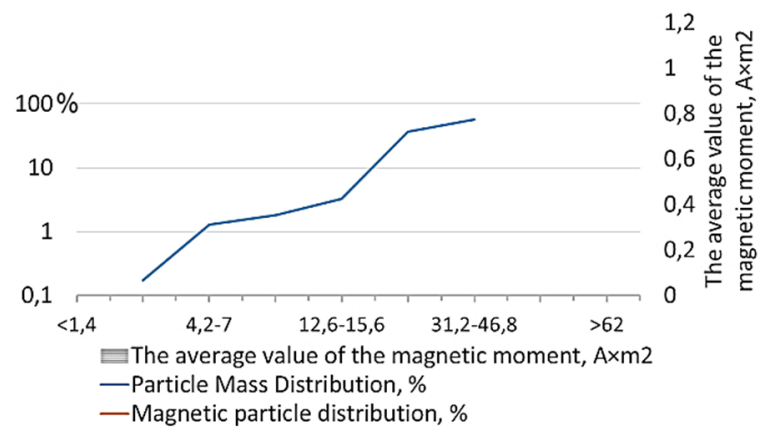

Fig. 5. The disperse and magnetic properties of the not magnetized dust from the scrubber generated by magnetizing roasting of the ore

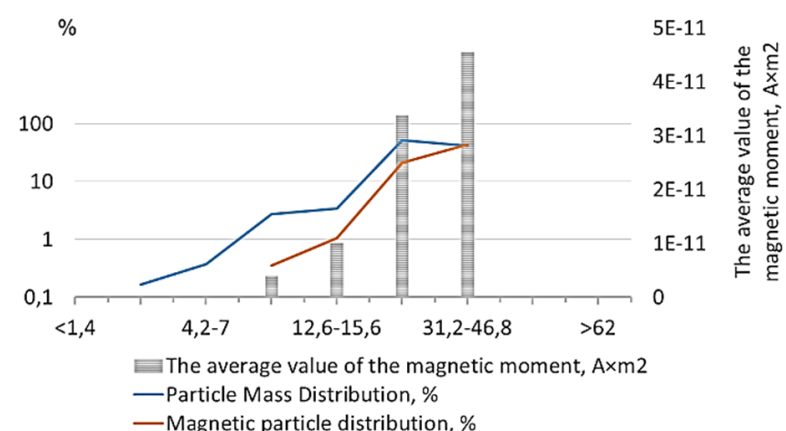

Fig. 6. The disperse and magnetic properties of the not magnetized dust from the scrubber generated by magnetizing roasting of the ore

The dust sampled from the scrubber has no initial magnetization. Because in the dust of the scrubber, there is a higher number of small particles, the temperature of which in the furnace passes through the Curie point. In this case, demagnetization occurs. Large particles have higher thermal inertia and, therefore, do not always have time to demagnetize. Small particles have a large specific surface, therefore, in the process of magnetizing firing, they are better restored, and their magnetic properties increase more. This feature explains the sizeable final magnetization of dust taken from the scrubber.

The studies of the characteristics of the dust generated during transportation of concentrate used the dust sampling from the cyclone hopper. In Fig. 7 and 8 show the results of studies of the dispersed and magnetic properties of dust.

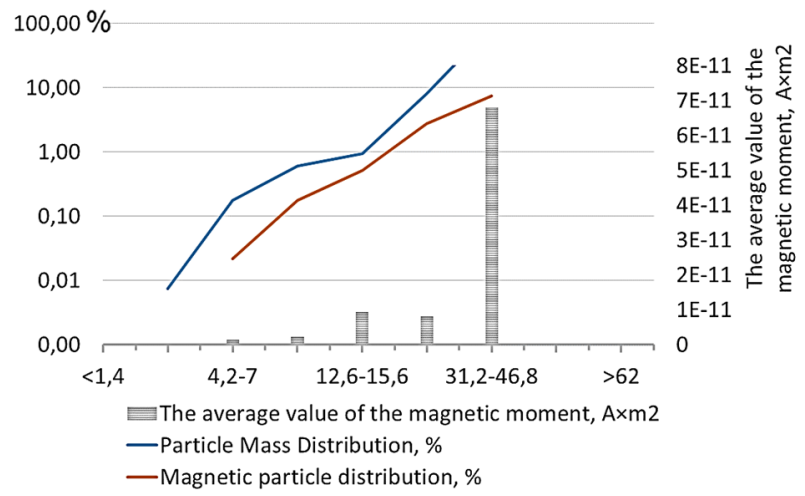

Fig. 7. The disperse and magnetic properties of the not magnetized dust of concentrate

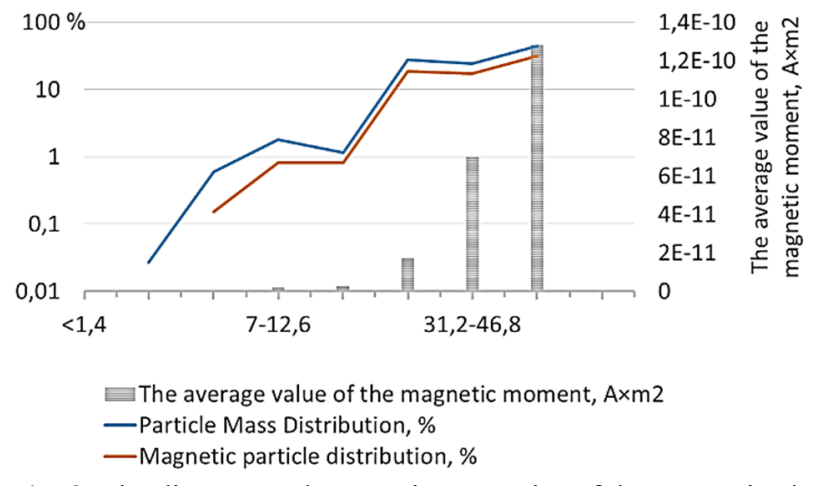

Fig. 8. The disperse and magnetic properties of the magnetized dust of concentrate

An analysis of the results shows that a significant proportion of the magnetic substance in the dust of concentrate is $97 \%$ versus $42 \%$ for the dust from the kiln. This circumstance, as well as the fact that the enriching of the ore is by strong magnetic fields, determines the high initial and final magnetization of this type of dust.

The studies of the characteristics of the dust generated during the firing of pellets used the dust sampling from the outer hatches of the cyclone hopper. Dust formed because of the impacts of the pellets against each other and the wall of the kiln. Therefore, a higher number of finely dispersed particles with significant magnetization and initial magnetization appear. The research results are shown in Fig. 9 and 10. An analysis of the results shows that a high average dust magnetization $(4.5 \mathrm{kA} / \mathrm{m})$ created by large fractions (23.4-54.6 $\mu \mathrm{m})$. However, particles less than $15.6 \mu \mathrm{m}$ in size have the greatest magnetization (up to $30 \mathrm{kA} / \mathrm{m}$ ). A difference in the magnetization of the particles of the $2.8 \mu \mathrm{m}$ fraction in the magnetized and non-magnetized state noted this is because of the temperature transition of these particles during firing through the Curie point.

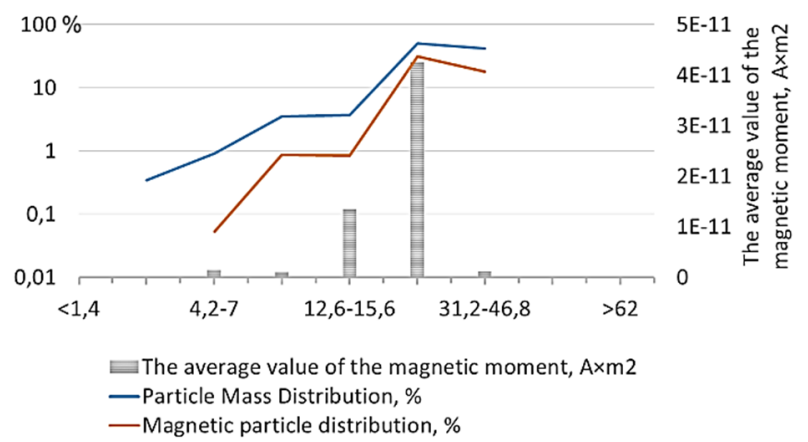

Fig. 9. The disperse and magnetic properties of the not magnetized dust generated during the firing of pellets

The studies of the characteristics of the dust of the sinter machine used the dust sampling from the cyclone hopper, as well as from electrostatic precipitators during the preventive stop of the sinter machine. In Fig. $11-14$ show dust research results.

A comparison of the results shows that the dust selected from the III zone of electrostatic precipitators has the smallest average size and the largest residual magnetization. From cyclone to zone III, the fraction of magnetized particles, as well as their remanent magnetization, increases in each dust fraction. From the 
cyclone to III in each zone increases the proportion of magnetized particles fractions of dust and their remanence. This feature explained by the fact that when moving at a higher speed in an inhomogeneous electric field, the particles experience the action of a magnetic field. Because of this, the magnetization of dust occurs.

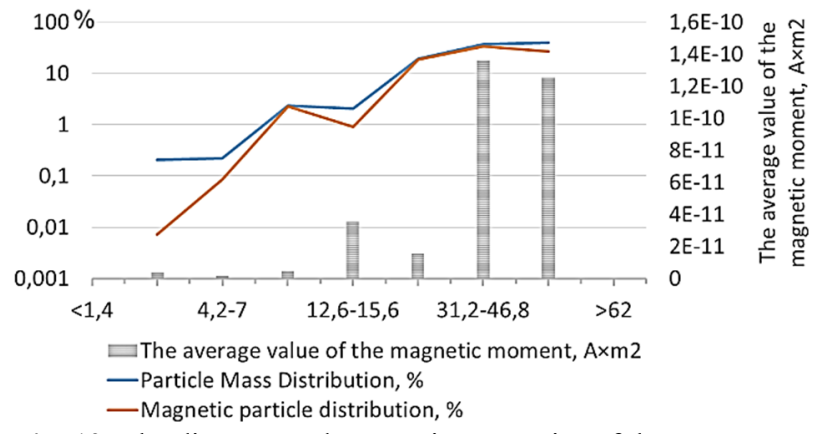

Fig. 10. The disperse and magnetic properties of the not magnetized dust generated during the firing of pellets

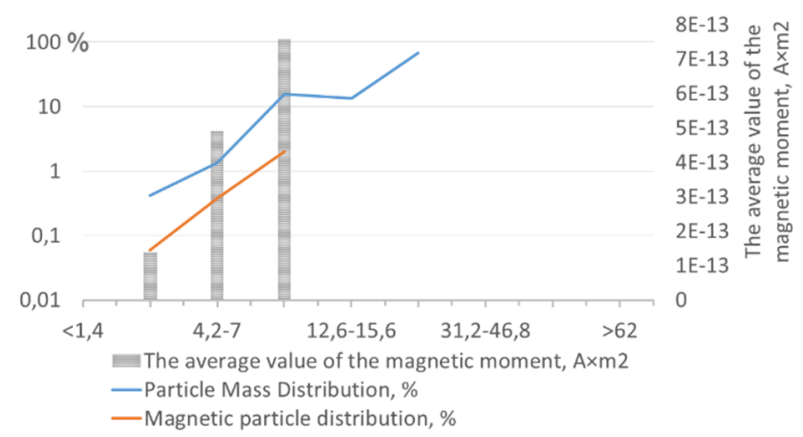

Fig. 11. The disperse and magnetic properties of the dust of the sintering machine from cyclone

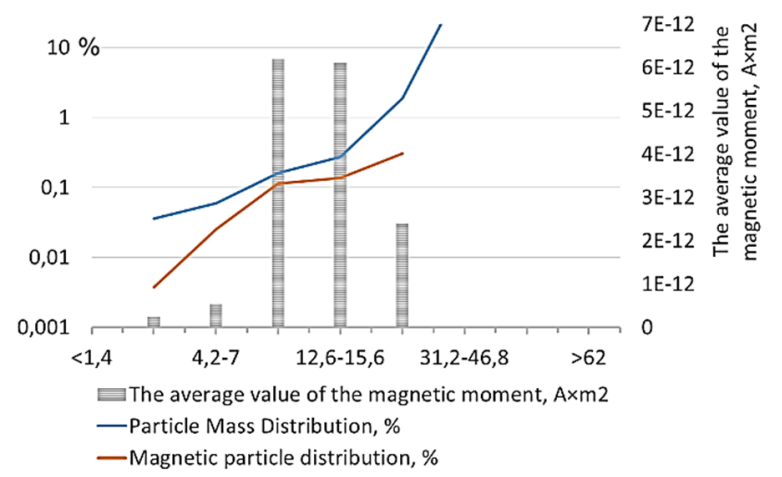

Fig. 12. The disperse and magnetic properties of the dust of the sintering machine from electrostatic precipitator zone I

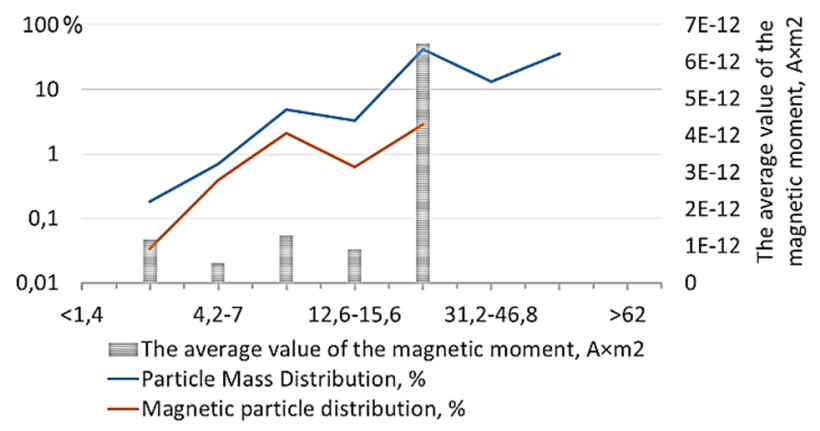

Fig. 13. The disperse and magnetic properties of the dust of the sintering machine from electrostatic precipitator zone II

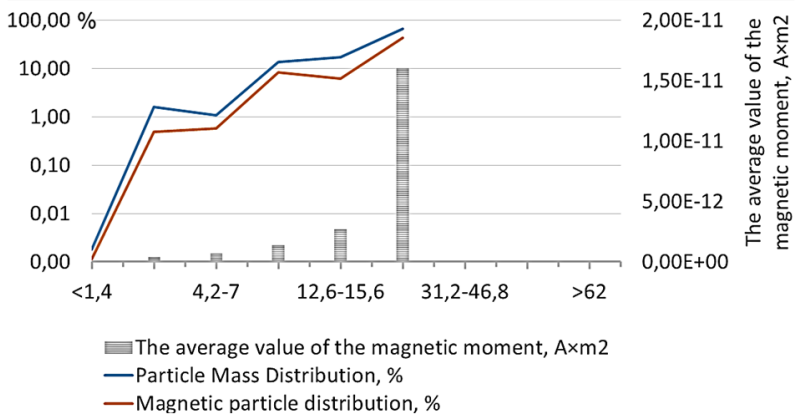

Fig. 14. The disperse and magnetic properties of the dust of the sintering machine from electrostatic precipitator zone II

In Fig. 15 - 16 shown the comparative characteristics of all investigated dust. It is known that when mixing solids, the uniformity of the mixture increases with a decrease in the size of its constituent particles. Most of the studied dust consists of particles with a size of $31.2-62.0 \mu \mathrm{m}$ (Fig. 3). Only for agglomeration dust from the III zone of the electrostatic precipitator, the bulk of the particles have a size of 12.6-31.2 $\mu \mathrm{m}$. When mixed with cement, this dust allows getting the most uniform distribution of particles in the binder.

Researches show that after magnetization in the dust, the proportion of largess particles increases due to smaller ones. The study of this phenomenon showed that this is due to the aggregation of magnetized particles.

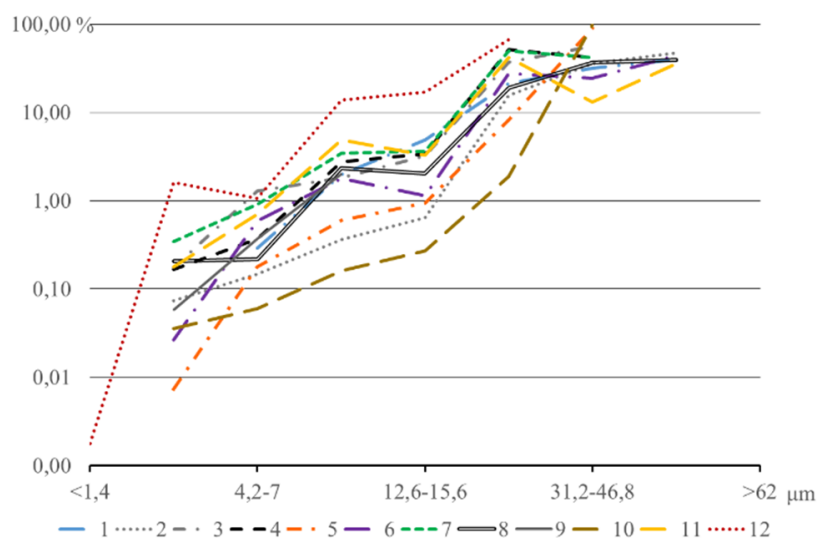

Fig. 15. Fractional composition of the dust. Dust numbers correspond to table 5 .

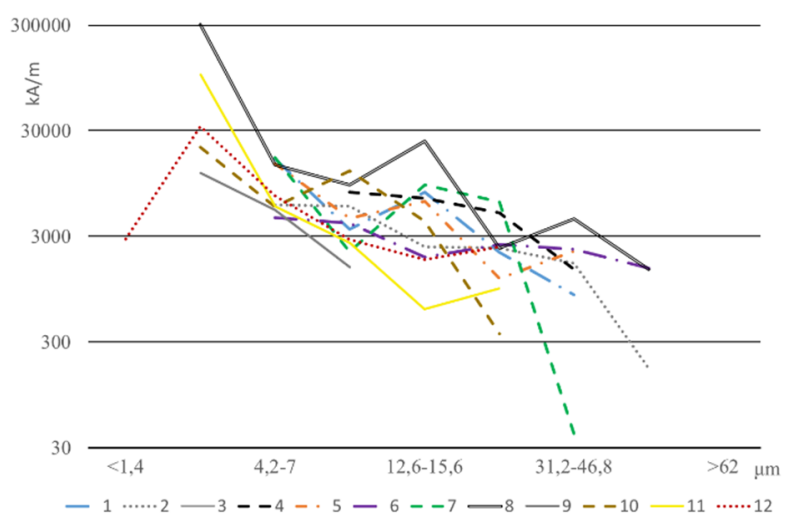

Fig. 16. The average value of the magnetization of dust by fractions, $\mathrm{kA} / \mathrm{m}$. Dust numbers correspond to table 5 .

The magnetic field strength decreases in proportion to the distance from the source. Calculations show that an 
increase in the size of dust particles at a constant concentration in the binder increases the distance between the dust particles, the sources of the magnetic field. Increases of the distance create an uneven magnetic field inside the binder and, accordingly, an inhomogeneous activation effect. On the contrary, a decrease in the size of dust particles leads to a decrease in the distance between the magnetic field sources and an increase in the quality of the activation effect. The majority of particles with magnetic properties in most dust have a size of $31.2-$ $62.0 \mu \mathrm{m}$.

The most significant part of the magnetic particles in the dust of the sintering machine has a size of $12.6-$ $31.2 \mu \mathrm{m}$, and the fraction $1.4-12.6 \mu \mathrm{m}$ from the III zone of the electrostatic precipitator accounts for more than $13 \%$ of all magnetic particles. This fact allows us to conclude that using the dust of the sintering machine from zone III of the electrostatic precipitator as an activating additive can be achieved the highest uniformity of magnetic fields in cement.

The methodology used to study the properties of dust used in this work makes it possible to determine the contribution of each fraction to the creation of a magnetization. Studies have shown that the magnetization of dust particles increases with decreasing particle size. The dust is polymineral and contains in its composition both components with and without magnetic properties. During particle fragmentation, the destruction occurs primarily along the cleavage planes of grains having differences in magnetic properties. As a result, either particle having high magnetic characteristics or not having them at all formed.

It is characteristic of the studied dust that with a decrease in the size of the fractions, the fraction of dust particles with magnetic properties decrease. However, the large magnetization of fine dust is the reason that a decrease in the proportion of magnetic particles does not correspond to a decrease in magnetization. For example, in agglomeration dust from the III zone of electrostatic precipitators, the proportion of particles with a size of $1.4-12.6 \mu \mathrm{m}$ having magnetic properties is $5.2 \%$ of the total mass of dust, and their contribution to the total magnetization is $15.3 \%$.

In most dust, particles of $12.6-62.0 \mu \mathrm{m}$ is made the main contribution to the creation of magnetization (Fig. 4).

Dust of the sintering machine from electrostatic precipitator zone III creates the bulk of the magnetization by particles of $1.4-31.2 \mu \mathrm{m}$ in size, which is a prerequisite for obtaining a high activation effect.

The averaged values of the magnetic and dispersed properties of the dust required for further studies shown in Fig. 17 and 18.

If the mean diameter of dust particles, their mass and mass fraction in the mixture is known, assuming that the particles located in the mix at the same distance, we can calculate the average length, $r_{\text {mean }}$ between dust particles in the mixture with cement.

In total, were studied 12 dust with various combinations of magnetic and dispersed characteristics. Since other characteristics of dust differ little from each other, the obtained results allow us to conduct studies to identify the general laws of the influence of various dust on the activation of a binder.

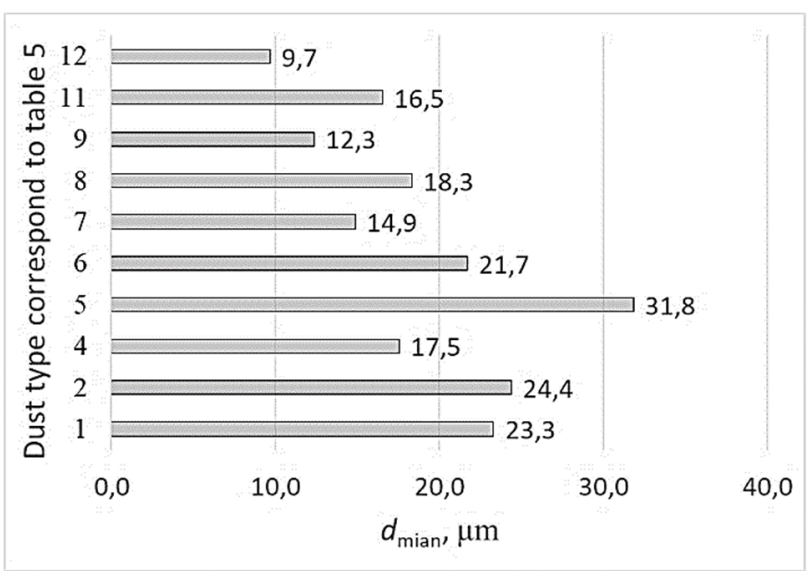

Fig. 17. The mean diameter of different types of dust.

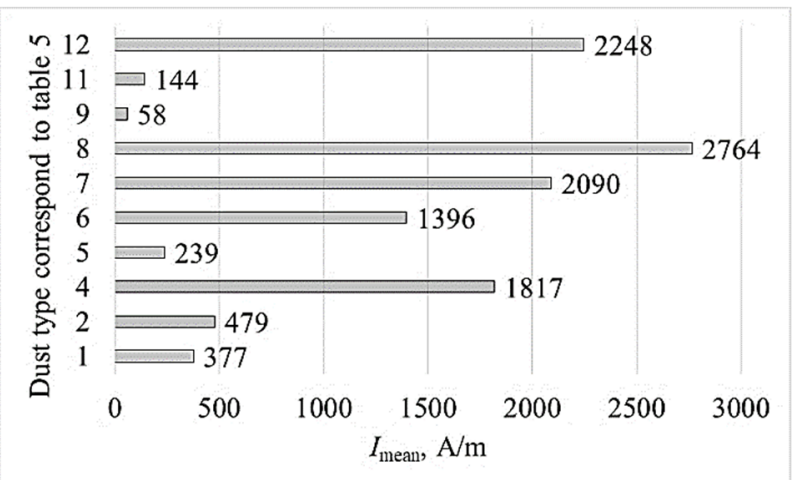

Fig. 18. The mean magnetization of different types of dust.

The average diameter of the largest of the studied dust is 2.5 times larger than that of the smallest. The minimum and maximum values of the average magnetization of different dust differ by more than 40 times.

Processing of the obtained data has shown the relationship between the activity of binders and the magnetic and dispersed characteristics of the introduced additives. In studying were investigated the strength characteristics of cement-sand mortars made from binders containing $98 \%$ of blast furnace cement and $2 \%$ of one of the dust. Mixing cement with the additive was carried out in a ball mill for 8 minutes. Further tests were carried out by DSTU EN 196-1:2007. In table 6 and Fig. $19-20$ show the test results.

Processing of the results showed that the increase in strength correlates with the average distance between particles and the magnetization of dust. Dependencies are:

$$
\begin{aligned}
& R_{m}=5,376+0,0012\left(\frac{I_{\text {mean }}}{r_{\text {mean }}}\right) \\
& R_{c}=37,84+0,028\left(\frac{I_{\text {mean }}}{r_{\text {mean }}}\right)
\end{aligned}
$$

Correlation coefficients for $(1)-K_{\text {cor }}=0.913$ with a standard error of 0.07 , for (2) $-K_{\text {cor }}=0.911$ with a standard error of 1.7 .

Reducing the size of dust particles affects the activation effect in two ways: firstly, the uniformity of mixing of particles increases, which improves the uniformity of magnetic fields induced by dust, and 
secondly, with decreasing particle size, their magnetization increases, which also increases the activity of binders.

Table 6. The dependence of the characteristics of cement-sand mortars on the properties of the introduced iron dust.

\begin{tabular}{|c|c|c|c|c|}
\hline Type of input dust & $\begin{array}{c}\text { The mean } \\
\text { distance } \\
\text { between } \\
\text { particles } \\
\text { in the } \\
\text { mixture } \\
r_{\text {mean }}, \mu \mathrm{m}\end{array}$ & $\frac{I_{\text {mean }}}{r_{\text {mean }}}$ & $\begin{array}{l}R_{\mathrm{m}} \\
\mathbf{M P a}\end{array}$ & $\begin{array}{l}R_{\mathrm{c}} \\
\mathbf{M P a}\end{array}$ \\
\hline No additives & & & 5.26 & 33.45 \\
\hline $\begin{array}{l}\text { Not magnetized from } \\
\text { the collector }\end{array}$ & 14.8 & 25.5 & 5.42 & 37.36 \\
\hline $\begin{array}{l}\text { Magnetized from the } \\
\text { collector }\end{array}$ & 15.4 & 31.0 & 5.46 & 38.39 \\
\hline $\begin{array}{l}\text { Magnetized from the } \\
\text { scrubber }\end{array}$ & 11.1 & 163.5 & 5.52 & 42.22 \\
\hline $\begin{array}{l}\text { Not magnetized dust of } \\
\text { concentrate }\end{array}$ & 20.2 & 11.9 & 5.31 & 39.2 \\
\hline $\begin{array}{l}\text { Magnetized dust of } \\
\text { concentrate }\end{array}$ & 13.8 & 101.4 & 5.54 & 43.67 \\
\hline $\begin{array}{l}\text { Not magnetized dust of } \\
\text { the sintering machine }\end{array}$ & 9.4 & 221.8 & 5.52 & 42.22 \\
\hline $\begin{array}{l}\text { Magnetized dust of the } \\
\text { sintering machine }\end{array}$ & 11.6 & 238.0 & 5.75 & 46.92 \\
\hline From cyclone & 7.8 & 7.4 & 5.37 & 36.72 \\
\hline $\begin{array}{l}\text { From electrostatic } \\
\text { precipitator zone II }\end{array}$ & 10.5 & 13.7 & 5.43 & 37.95 \\
\hline $\begin{array}{l}\text { From electrostatic } \\
\text { precipitator zone III }\end{array}$ & 6.1 & 366.9 & 5.81 & 47.34 \\
\hline
\end{tabular}

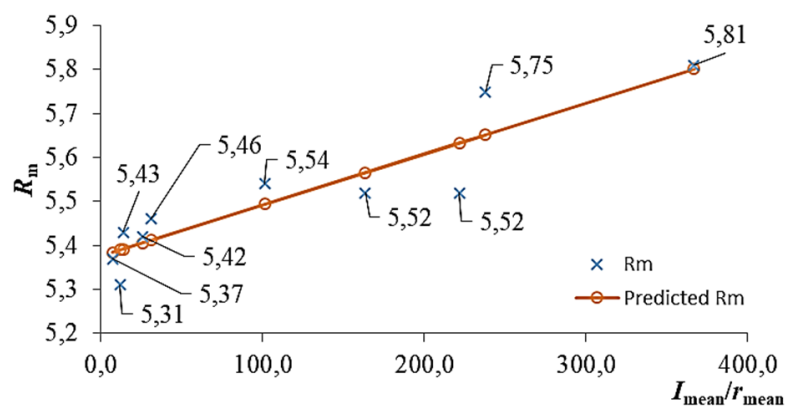

Fig. 19. Effect of dust characteristics on cement bending strength.

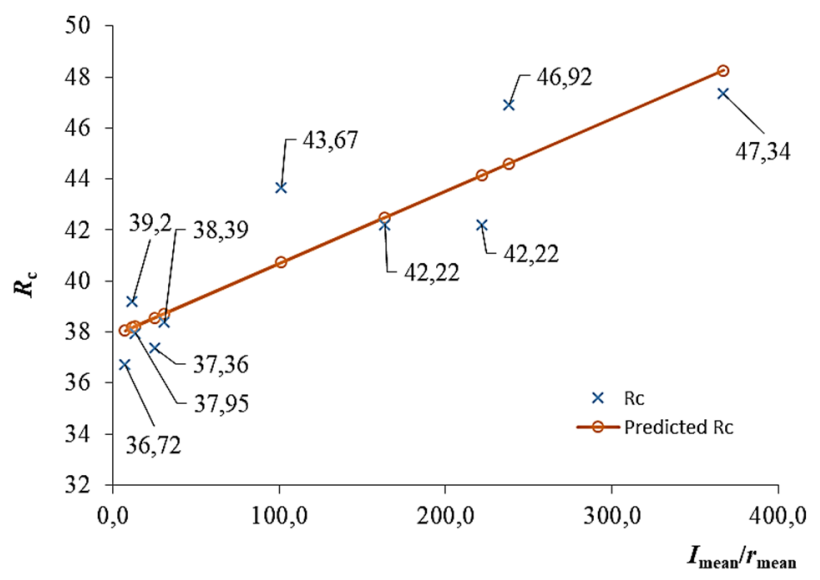

Fig. 20. Effect of dust characteristics on cement compressive strength
It follows that the most significant activation effect achieved by introducing iron-containing magnetized dusty substances into the binder, in which the ratio $I_{\text {mean }} / r_{\text {mean }}$ is of the highest importance. Among those considered, this is the dust of the sintering machine taken from electrostatic precipitator zone III. Its activation effect, along with magnetized dust from the kiln, is $41 \%$. However, unlike the latter, dust from the III zone of the electrostatic precipitator does not need magnetization.

\section{Conclusions}

The studies show that the magnetic characteristics determined by the origin and method of dust collection. Studies revealed that with decreasing dust particle size, their magnetization increases. After additional magnetization, the fractional composition of dust changes due to the aggregation of small parts into large ones.

The studied dust has 12 different combinations of magnetic and dispersed characteristics, which is enough to identify the patterns of the influence of each of the factors on the activity of binders.

In studies was established that from the studied dust, the highest uniformity of magnetic fields in the binder allows getting dust from the III zone of the sinter machine electrostatic precipitators.

The investigation established that the introduction of pre-magnetized ferromagnetic dust into the composition of blast furnace cement has an activating effect on the process of binder hydration.

Studies proved that the degree of influence of the additive on the binder activity depends on the distance between the dust particles and the magnetic field strength.

In the future, it is necessary to study the processes of structure formation under the influence of a ferromagnetic additive and their influence on the strength and deformation characteristics of mortar and concrete.

\section{References}

1. L. Lapcik, Z. Simek, Electron paramagnetic resonance study of dry cements. Cem. Concr. Res. 26(2), 237 (1996)

2. E. Lopanova, Radiospectroscopic researches of process of hydration of silicates with the help of spin labels. Voprosy Materialovedeniya 3, 34 (2004)

3. D. Afanas'ev, L. Tsyro, A. Unger, L. Andreeva, S. Alexandrova, F. Unger, Spin aspects in the nature of cement hardening. Polzunovsky Vestnik 3, 82 (2009)

4. D. Afanas'ev, L. Tsyro, A. Unger, L. Andreeva, S. Alexandrova, F. Unger, Spin chemistry of cement systems. Vestnik nauki Sibiry 5, 247 (2012)

5. D. Afanas'ev, F. Unger, L. Tsyro, Y. Sarkisov, N. Gorlenco, The role of spin effects in structure formation of cement mixtures. Vestnik TGASU 2, 94 (2014) 
6. A. Buchachenko, Second generation of magnetic effects in chemical reactions. Russ. Chem. Rev. 62, 1139 (1993)

7. V. Pomazkin, A. Makaeva, The magnetic-activated water in the building technologies. Vestnik OSU 1, 109 (2001)

8. N. Nechistyak, I. Stepanchikova, RU Patent 2678749. Izobreteniya. Poleznye modeli 4 (2019)

9. I. Podkovyrkov, Influence of magnetic fields on matter, including on building materials. Aktual'nye problemy sovremenoj nauki 3, 242 (2015)

10. V. Selyaev, V. Kolotushkin, Influence of technological modes of magnetic activasion on elastic strength characteristics of cement composites. Regional Architecture and Engineering 2(27), 17 (2016)

11. A. Berezjnoy, V. Kovalenko, USSR Patent 291892. Otkrytiya. Izobreteniya 4, 64 (1971)

12. E. Smolin, V. Kulsartov, V. Zelenkov, USSR Patent 833739. Otkrytiya. Izobreteniya 20, 73 (1981)

13. S. Shinkorenko, E. Beletsky, A. Shiryaev, Spravocnik po obogascheniyu rud chernykh metallov (Ferrous Ore Enrichment Guide). (Nedra, Moscow, 1980)

14. V. Derkach, Specialnye metody obogascheniya poleznyh iskopaemykh (Special Mineral Processing Methods). (Nedra, Moscow, 1966)

15. S. Andonyev, O. Filipiev, Pylegazovye vybrosy predpriyatiy chernoy metallurgiyi. (Dust and gas emissions from ferrous metallurgy enterprises). (Metallurgiya, Moscow, 1973) 